\title{
Active Galactic Nuclei at the Crossroads of Astrophysics
}

\author{
Andrei Lobanov and J. Anton Zensus \\ Max-Planck-Institut für Radioastronomie, Auf dem Hügel 69, 53121 Bonn, Germany
}

\begin{abstract}
Over the last five decades, AGN studies have produced a number of spectacular examples of synergies and multifaceted approaches in astrophysics. The field of AGN research now spans the entire spectral range and covers more than twelve orders of magnitude in the spatial and temporal domains. The next generation of astrophysical facilities will open up new possibilities for AGN studies, especially in the areas of high-resolution and high-fidelity imaging and spectroscopy of nuclear regions in the X-ray, optical, and radio bands. These studies will address in detail a number of critical issues in AGN research such as processes in the immediate vicinity of supermassive black holes, physical conditions of broad-line and narrow-line regions, formation and evolution of accretion disks and relativistic outflows, and the connection between nuclear activity and galaxy evolution.
\end{abstract}

\section{Nuclear activity in galaxies}

Recent years have witnessed substantial progress in studies of active galactic nuclei (AGN). Activity is observed in galaxies throughout the entire range of the electromagnetic spectrum [35]. It is manifested on spatial and temporal scales that span over 12 orders of magnitude, ranging from several Schwarzschild radii to megaparsecs and from minutes to millions of years [141]. On the surface, the activity of galactic nuclei comes in many faces, outlined by the uncomfortably large number of the AGN classes - yet the underlying mechanism and physical conditions in the nuclear regions of all galaxies are probably more similar than may appear at first glance $[4,53,178]$. Last but not least, AGN are now detected at redshifts of 6 and beyond [40], and they may be closely connected to the formation and evolution of the large-scale structure [124] and to the epoch of reionization of the Universe $[39,113,151]$.

There is growing acceptance of ubiquity of the nuclear activity in galaxies and its connection to the presence of supermassive black holes (SMBH) in the centres of all massive galaxies [3,57,74,75]. Observations in the infrared [52] and sub-millimetre bands [124] indicate that almost every galaxy exhibits certain characteristics previously thought to be related only to a much narrower class of powerful AGN. This implies that virtually all massive galaxies may go through a strong AGN phase in the course of their cosmological evolution. Historically, observational studies of AGN have been concentrated largely within six broadly defined areas.

1. Surveys have been used effectively, first in the optical and radio domains and later throughout much of the electromagnetic spectrum, to find correlations 
between different types of AGN and to trace their cosmological evolution. The databases provided by the ROSAT, IRAS, ISO, SIRTF and VLA (FIRST [7] and NVSS [21]) surveys have become the true cornerstones of AGN research, as well as the HST and Chandra deep field observations. These efforts will be taken further by the ongoing SDSS survey [1] and the Spitzer surveys [41]. The Spitzer data have already yielded a fundamental result on the obscuration in AGN, indicating that as much as three quarters of all AGN are likely to be obscured at all redshifts [176], which implies that the number of active galaxies may be much higher than was thought. AGN studies in the next decade will also benefit from multi-wavelength surveys such as the great observatories origins deep survey (GOODS [56]) which addresses AGN structure and demographics [66], unification scheme and and AGN-SMBH co-evolution [53,176]. A number of ground and space VLBI surveys undertaken in the radio in the centimetre $[43,47,70,72,88,98,137,143,168-170,186]$ and millimetre $[109,110,149]$ domains have revealed a wealth of information about morphology $[76,94,193]$, kinematics [87] and evolution of radio-emitting material in the nuclear regions and relativistic outflows in AGN on scales down to fractions of a parsec. VLBI surveys have also been used effectively for addressing a variety of general astrophysical problems including AGN evolution [161] and population modelling [97,100], jet formation [109], fundamental astrophysical emission processes [86,99], and cosmology [60].

2. Morphological studies of AGN have been done extensively in the radio [84,136], optical [139] and X-ray regimes [118,119], recovering large-scale structures produced by AGN. Circumnuclear disks and tori have been revealed conclusively in recent optical, near-infrared and radio observations $[79,91,128,183]$ (Fig. 1). Most spectacularly, recent X-ray images obtained with Chandra have shown the shocks and ripples in the intergalactic medium excited by the central engine in NGC 1275 [38].

3. Variability studies of long- and short-term changes of continuum and line emission have enabled detailed investigations of non-stationary processes in the immediate vicinity of the SMBH and understanding better the physics of nuclear regions of AGN [174]. Timescales probed by these studies range from hours to several decades $[2,6,141,159,165,181,194]$. The variable continuum flux is believed to be responsible for ionizing the cloud material in the broad-line region (BLR). Optical spectral line and continuum variability data have been combined to model the BLR $[83,141,142]$, which provided a reliable method for estimating the mass of the central black holes in a number of AGN [141]. In radioquiet AGN, short-term variations of the continuum X-ray emission $[5,14]$ are most likely connected with processes occurring the accretion disk at distances of $\sim 10-100 R_{\mathrm{g}}\left(R_{\mathrm{g}}=G M_{\mathrm{bh}} / c^{2}\right.$ is the gravitational radius for a black hole of mass $M_{\mathrm{bh}}$, where $G$ is the Newtonian gravitational constant) from the black hole [130]. This conclusion is contested by multi-wavelength monitoring observations $[24,141]$ showing no apparent time delay between flux variations in the ultraviolet (UV) and optical continua, which indicates that instabilities in the accretion disk or random fluctuation in the accretion rate alone cannot explain 


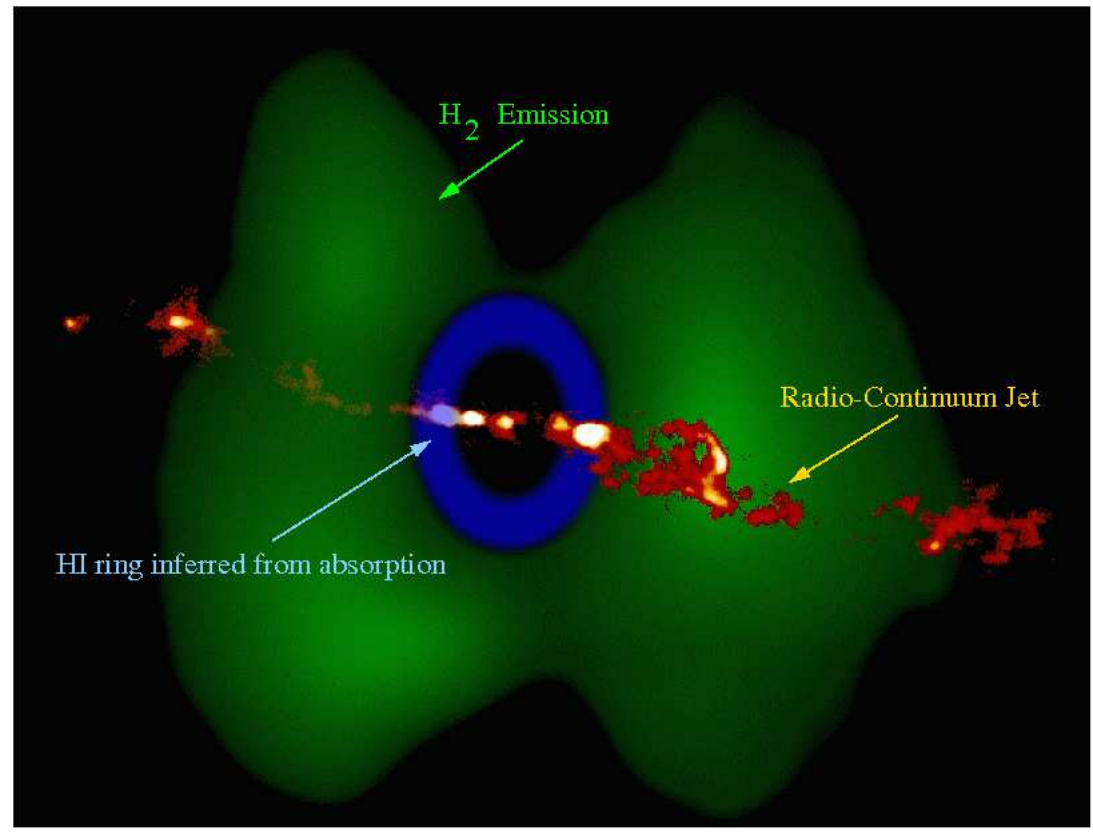

Fig. 1. Montage of the inner 250 pc of NGC 4151. The $\mathrm{H}_{2}$ emission traces a torus, the $\mathrm{H}$ I absorption comes from a ring inside the torus. Ionized gas (black) is assumed to fill the torus inside the $\mathrm{H}$ I ring [128].

the continuum variability [141]. In radio-loud AGN, continuum emission from the relativistic plasma in the jet dominates at all energies $[175,185]$, swamping the X-ray emission associated with the accretion flow. Hence, the continuum variability in radio-loud AGN may be related to both the jet and the instabilities of accretion flows $[130,175]$ near the central engine.

Long-term variability of X-ray $[119,126,160]$ and radio emission [2] probably reflects instabilities developing in the disk [105] and shocks propagating in the relativistic jet [45]. Ejections of new jet components have been reported to occur after minima in the X-ray light curve [119], suggesting that the jet is fed by the material falling onto the black hole from inner radii of the accretion disk. No observational evidence has been reported so far for a link between optical/UV continuum variability and the compact radio jet.

Intraday variations observed in the radio [95,180], optical [67], IR [59], and Xray domains [5] most likely reflect processes in the immediate vicinity of the central engine and in internal shocks developing in relativistic outflows [156,162,163]. In this respect, intraday variability in AGN may be similar to quasi-periodic oscillations observed in Galactic X-ray binaries. Interstellar scintillations are also likely to play a role in the intraday variations observed in the radio regime $[10,150]$.

4. Multifrequency campaigns, albeit proven difficult to organize, have yielded accurate broad-band spectra of AGN $[116,172]$ and have enabled detailed in- 
vestigations of physical processes governing the production of the non-thermal continuum emission from circumnuclear regions in AGN [64,173]. Multifrequency data were used to determine the spectral energy distribution in AGN in the quiescent and flaring stages $[48,121,158]$ and to characterize the properties of the synchrotron emission in relativistic outflows $[26,102]$.

5. Spectroscopic studies in the optical $[8,58,120,184]$ infrared [111] radio [104], and increasingly also in the X-ray domain $[34,37,115,131,132,135,157,167,187]$ have provided measurements of the gas and stellar kinematics near the central black hole [189], in the broad and narrow line regions of galactic nuclei. These studies will be greatly enhanced with the systematic spectroscopy provided by the SDSS data and with the high-energy spectroscopy data from the existing and planned X-ray missions.

6. Dedicated monitoring programs have been employed successfully virtually in all observational domains to study in detail the dynamics and physical conditions in the nuclear regions of a number of prominent AGN. Structure of the nuclear regions has been imaged extensively with high resolution radio interferometry, uncovering the evolution of radio emitting plasma on linear scales from $\approx 100 R_{\mathrm{g}}[80,96]$ to $\approx 1 \mathrm{kpc}[182,191,192]$. Radio monitoring programs are now reaching timescales of several decades $[22,106,105]$. Radio frequency observations of maser emission have been used to probe the presence of accretion disks $[71,127]$ and molecular tori [90] around putative black holes in the centres of AGN. Extragalactic maser emission has provided direct evidence of interaction between the dense molecular material and the ionization cones [50] or nuclear jets $[20,138]$. Properties of relativistic outflows have been connected to the physical conditions in the nuclear regions $[81,101]$ and used for identifying possible binary systems of SMBH in AGN $[19,105,152]$.

High-resolution radio observations of Seyfert galaxies have offered an opportunity to study the optical emission from the immediate vicinity of the central engine of AGN, on scales comparable to the largest extent of the BLR. Optical observations of broad emission lines have been instrumental for understanding the structure and dynamics of the circumnuclear gas, and they have yielded an attractive concept of a disk-like morphology of the BLR [146]. The detection and modelling of several double-peaked Balmer lines has further supported this idea. In most cases, the double-peaked emission lines can be explained with line emission that originates in the disk [33]. There are, however, two very notable exceptions among the radio-quiet AGN. The core of the broad double-peaked emission lines in RX J1042+1212 [148] and Ark 120 [145] is most likely to originate from an outflow-related component of the BLR (Fig. 2). It is possible that this component is indeed related to an interaction between the relativistically moving plasma and high-velocity clouds situated in a sub-relativistic outflow/wind surrounding the jet $[32,129,147]$. In this case, the broad-line emitting region associated with the jet/outflow can be located at a significant distance from the nucleus, perhaps as large as $\sim 1 \mathrm{pc}$. Verifying the existence of the outflow-related component of the BLR and testing the putative connection between the BLR and relativistic outflows in Seyfert galaxies can therefore be viewed as criti- 

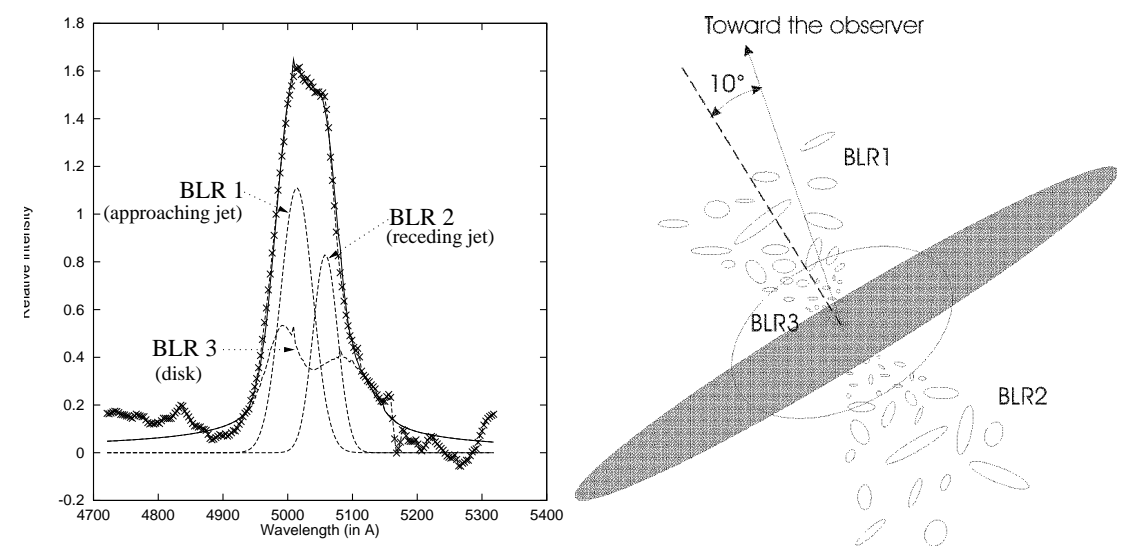

Fig. 2. Left: The observed $\mathrm{H}_{\beta}$ line of Ark 120 fitted by the two-component model (solid line): a disk (in the line wings) plus an outflow-related component of the BLR (the core of the line). The two components in the core correspond to the line emission generated in the approaching (left) and receding (right) part of a bipolar outflow. Right: The scheme of the proposed BLR model for Ark 120 [145].

cal experiments that may advance our understanding of the nuclear activity in galaxies.

\section{A synthetic view of AGN}

The nuclear environment in active galaxies hosts a variety of physical phenomena on scales ranging from $\sim 1-2 R_{\mathrm{g}}$ to $\sim 100$ parsecs. Table 1 lists most important components of the nuclear regions in galaxies. They cover over seven and ten orders of magnitude in linear and temporal scales, respectively. This presents a serious challenge to any attempt of creating a single framework for describing all aspects of nuclear activity. The key constituents of the nuclear environment can be divided into six broad categories:

Accretion disks and infalling material producing strong continuum and line emission in the high-energy (X-ray and possibly even $\Gamma$-ray) regime [5,78], and often exhibiting maser lines in the radio regime [71].

Broad-line and narrow-line regions detected in the line emission and absorption in the optical regime [141].

Obscuring torus composed of clouds of gas and dust [4] and manifested by emission and absorption, primarily in the optical [177], infrared [179] and radio domains [128].

Bipolar outflows detected in continuum emission throughout much of the electromagnetic spectrum. Sub-relativistic outflows are also manifested by the optical absorption lines (BAL outflows [32,49]) 
Table 1. Characteristic scales in the nuclear regions in active galaxies

\begin{tabular}{rccccc}
\hline \hline & $l$ & $l_{8}$ & $\theta_{\mathrm{Gpc}}$ & $\tau_{c}$ \\
{$\left[R_{\mathrm{g}}\right]$} & {$[\mathrm{pc}]$} & {$[\mathrm{mas}]$} & $\begin{array}{c}\tau_{\mathrm{orb}} \\
{[\mathrm{yr}]}\end{array}$ \\
\hline Event horizon: & $1-2$ & $10^{-5}$ & $5 \times 10^{-6}$ & 0.0001 & 0.001 \\
Ergosphere: & $1-2$ & $10^{-5}$ & $5 \times 10^{-6}$ & 0.0001 & 0.001 \\
Accretion disk: & $10^{1}-10^{3}$ & $10^{-4}-10^{-2}$ & 0.005 & $0.001-0.1$ & $0.2-15$ \\
Corona: & $10^{2}-10^{3}$ & $10^{-3}-10^{-2}$ & $5 \times 10^{-3}$ & $0.01-0.1$ & $0.5-15$ \\
Broad line region: & $10^{2}-10^{5}$ & $10^{-3}-1$ & 0.05 & $0.01-10$ & $0.5-15000$ \\
Molecular torus: & $>10^{5}$ & $>1$ & $>0.5$ & $>10$ & $>15000$ \\
Narrow line region: & $>10^{6}$ & $>10$ & $>5$ & $>100$ & $>500000$ \\
Jet formation: & $>10^{2}$ & $>10^{-3}$ & $>5 \times 10^{-4}$ & $>0.01$ & $>0.5$ \\
Jet visible in the radio: & $>10^{3}$ & $>10^{-2}$ & $>0.005$ & $>0.1$ & $>15$ \\
\hline
\end{tabular}

Column designation: $l$ - dimensionless scale in units of the gravitational radius, $G M / c^{2} ; l_{8}$ - corresponding linear scale, for a black hole with a mass of $5 \times 10^{8} \mathrm{M}_{\odot}$; $\theta_{\mathrm{Gpc}}-$ corresponding largest angular scale at $1 \mathrm{Gpc}$ distance; $\tau_{c}$ - rest frame light crossing time; $\tau_{\text {orb }}$ - rest frame orbital period, for a circular Keplerian orbit.

Nuclear stellar population detected in the optical through near IR regimes via velocity dispersion or individual stellar proper motions in the nearest AGN in $\operatorname{Sgr} \mathrm{A}^{\star}[31,54]$.

Secondary black holes in multiple black hole systems, which can be inferred from characteristic emission and structural variability in the $\mathrm{X}$-ray through radio regimes $[19,105,155]$.

The AGN studies outlined in the previous section have provided substantial knowledge about each of these aspects of nuclear activity. This has enabled the construction of a synthetic, "unified" picture of active galaxies, in which the entire spectrum of galactic activity is described as an intricate interplay between the physical conditions and orientation of individual constituents of an active nucleus $[4,53]$. In the current AGN paradigm, several key components, including the accretion disk, the broad-line region and the relativistic outflow (jet), play major roles [35], together with the putative central SMBH (Fig. 3). A fraction of the infalling material forms an outflow along the rotational axis of the SMBH $[11,12,45,129]$ and a strong, compact source of continuum radiation that ionizes the material in the BLR. The nature of the continuum source and its relation to the BLR and the jet remain unclear. The continuum source can be located in the accretion disk [46] or in the hot corona at 200-1000 $R_{\mathrm{g}}$ above the accretion disk [36,144]. A contribution from the jet cannot be excluded [42]. The BLR is often assumed to have an ellipsoidal shape, with at least a fraction of the BLR clouds interacting with the jet plasma. There is also growing evidence for the presence of a conical BLR component [145] associated with a slower, subrelativistic outflow originating in outer regions of the accretion disk [145]. The sub-relativistic outflow is believed to be responsible also for the broad absorption lines (BAL) observed in a number of quasars $[32,49]$. The disk, the BLR, and the outflows must be closely connected, producing the bulk of the AGN power. 


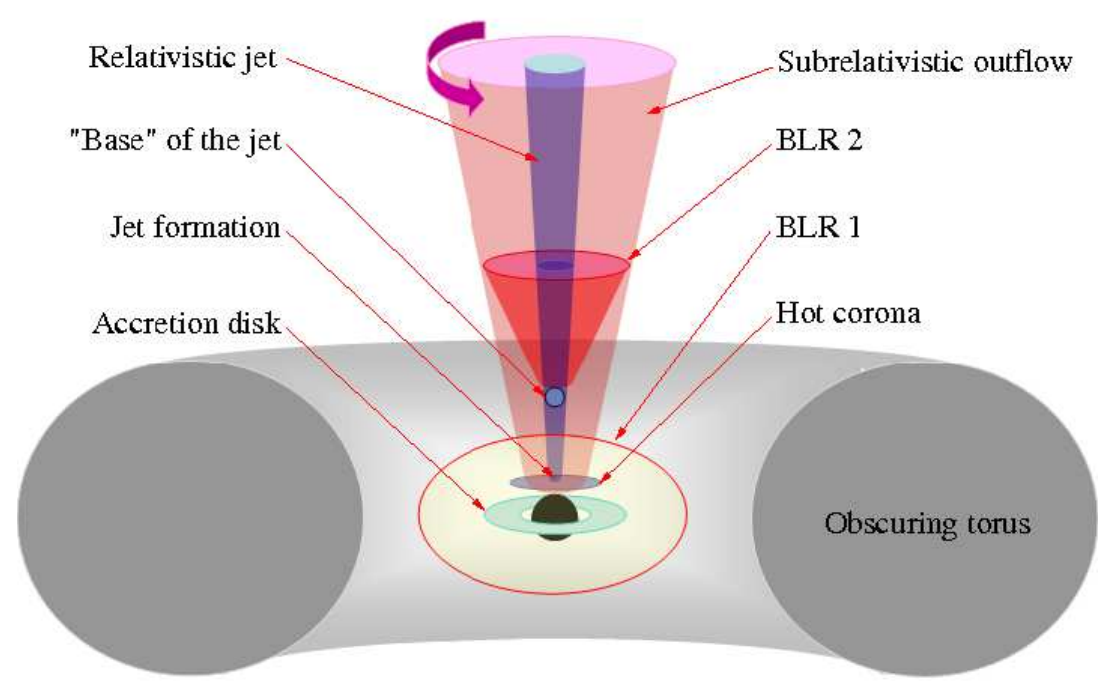

Fig. 3. A sketch of the nuclear region in an active galaxy (the drawing is made not to scale and shows only the approaching jet). The broad-line emission is likely to be generated both near the disk [141] (an ellipsoidal BLR 1, ionized by the emission from a hot corona $[36,144]$ or the accretion disk [46]) and in a rotating subrelativistic outflow $[32,129,147]$ surrounding the jet (a conically-shaped BLR 2, ionized by the emission from the relativistic plasma in the jet). BLR 2 is evident in the broad-line emission when the jet emission dominates the optical continuum. BLR 1 may be manifested in the broad-line emission when the jet contribution to the ionizing continuum is small.

Reconstructing the physical mechanism behind this connection is pivotal for understanding the AGN phenomenon in general.

\section{Zooming on the central engine}

The central engine, presumed to contain a SMBH, is an elusive formation. Direct detection and imaging of the event horizon of a black hole cannot be done with present instruments. The presence of SMBH in galactic centres has been inferred so far only on the basis of circumstantial evidence obtained from observations in the X-ray, optical, near infrared, and radio domains.

$X$-ray observations have revealed relativistically broadened line profiles indicative of motions at speeds exceeding $10^{5} \mathrm{~km} / \mathrm{s}$ (Fig. 4). The X-ray emission generated above the accretion disk interacts with the disk material and produces the iron fluorescence line $[37,38,126]$. Observed line profiles show relativistic speeds, Doppler shifts and gravitational redshift. Modelling of the line 

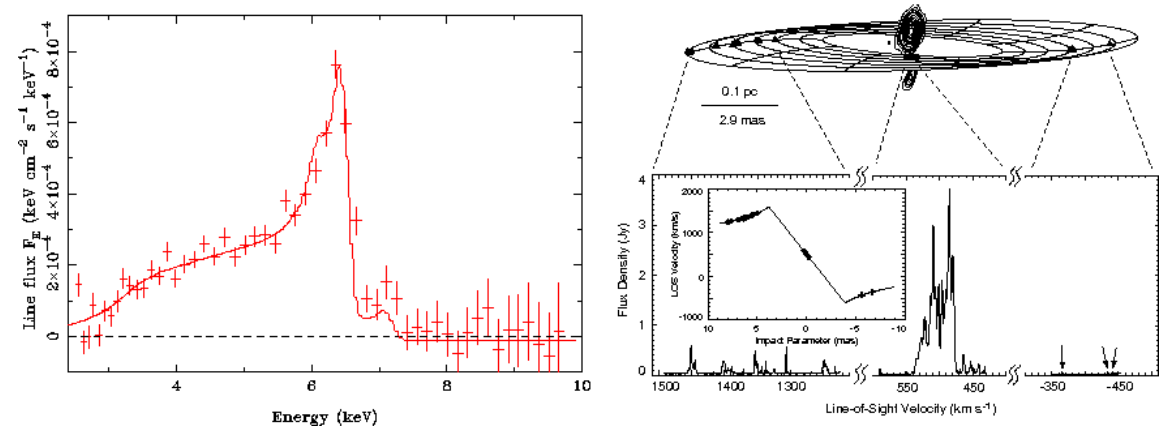

Fig. 4. Left: Relativistic Fe K $\alpha$ line profile in a Seyfert 1 galaxy MCG-6-30-15 obtained from a joint XMM-Newton and Beppo-SAX dataset [37]. The crosses mark the data points and the solid line marks the model in which the emission is generated in an accretion disk accretion and the inner radius of the emission is at $\approx 2 R_{\mathrm{g}}$. Right: $\mathrm{H}_{2} \mathrm{O}$ masers in NGC 4258. Top panel: actual maser positions (filled triangles and circles) superimposed on the radio-continuum emission (contours) and approximated by a model of warped disk. The filled square marks the best-fit location of the centre of the disk. Bottom panel: total spectrum of the maser emission and line of sigh velocities of individual spots fitted by a Keplerian disk model. The high-velocity masers trace a Keplerian curve to better than $1 \%$. Observations of $\mathrm{H}_{2} \mathrm{O}$ masers in NGC 4258 have allowed to infer a geometric distance of $7.2 \pm 0.3 \mathrm{Mpc}$ to the galaxy from the direct measurement of orbital motions in the maser spots. The motions imply a central object with a mass of $(3.9 \pm 0.1) \times 10^{7} \mathrm{M}_{\odot}[71]$.

profiles constrains the disk inclination and spin of the SMBH, which can be used to distinguish between rotating and non-rotating black holes [188].

Optical spectroscopy has revealed the Doppler shift caused by the fast Keplerian rotation of material in the accretion disk implying masses of $1.5 \times 10^{9} \mathrm{M}_{\odot}$ in $\mathrm{M} 84$ [13] and $2.5 \times 10^{9} \mathrm{M}_{\odot}$ in $\mathrm{M} 87$ [112].

Near-infrared observations of the nucleus of our own Galaxy show the proper motion of stars there [31,54]. This motion implies a mass of $\approx 3.7 \times 10^{6} \mathrm{M}_{\odot}$ enclosed within $45 \mathrm{AU}$ distance from the Galactic Centre [55].

Radio observations of maser lines in NGC 4258 (Fig. 4) have yielded the most accurate measure of distance and black hole mass in an external galaxy [71]. High-resolution radio observations of M 87 have probed directly scales as small as $100 R_{\mathrm{g}}[80,96]$. The presence of supermassive black holes in galactic centres is also implied indirectly by the exceptional stability of jet direction $[136,139]$ and apparent superluminal motions that require highly relativistic flows $[191,192]$.

\subsection{Physics of relativistic outflows from SMBH}

The activity of the central engine is accompanied by highly-relativistic collimated outflows (jets) of plasma material formed and accelerated in the vicinity of the black hole $[45,92,123]$. Inhomogeneities in the jet plasma appear as a series of compact radio knots (jet components) observed on scales ranging 


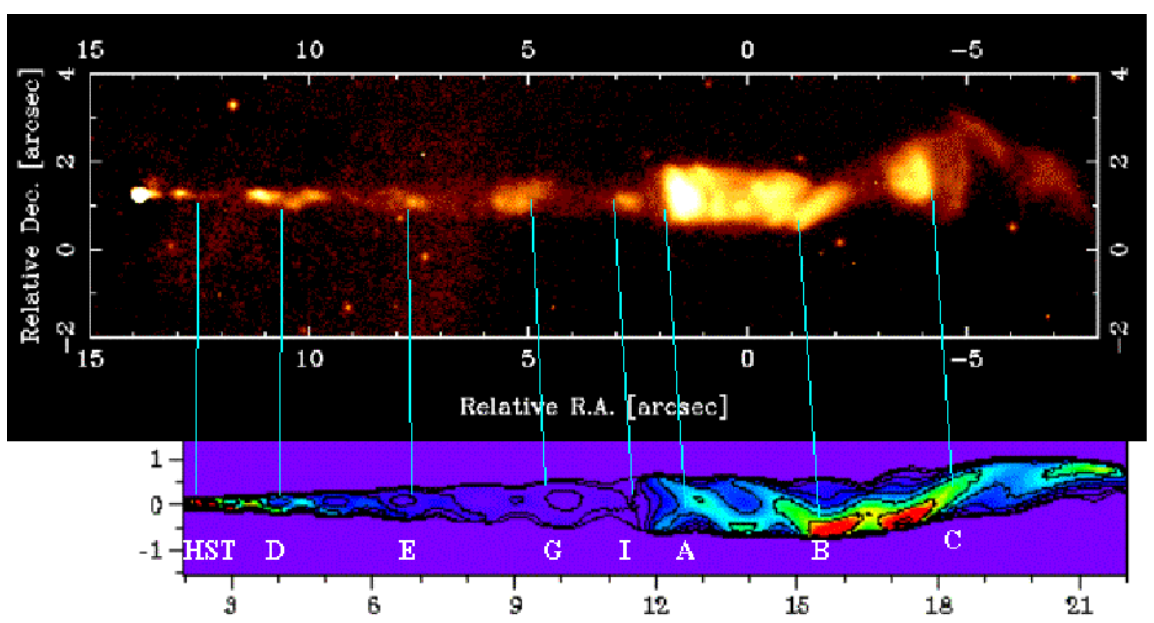

Fig. 5. Top: HST image of M 87 [139]. Botom: Line-of-sight synchrotron intensity image and contours at $\theta=40^{\circ}$ calculated from an analytical model of the internal structure of the jet using Kelvin-Helmholtz instability. Connecting lines illustrate the correspondence between the two images. The model represent well the brightness distribution in the jet on scales larger than $1 \mathrm{kpc}[108]$.

from several light weeks to about a kiloparsec [191,192]. The kinematics and spectral evolution of the knots are determined by relativistic shocks [106] and plasma instabilities $[102,107,108]$. Strong relativistic shocks tend to dissipate on scales of $\sim 10 \mathrm{pc}[106]$ and internal structure of large-scale jets is dominated by Kelvin-Helmholtz instability (Fig. 5). Close to the central engine, the kinematic and emission changes can reflect the dynamics in the central engine, and this can be used effectively for estimating the properties of the binary systems of SMBH $[19,105]$.

\section{$4 \mathrm{SMBH}$ in a larger context}

Supermassive black holes have now become the centrepiece of the modern AGN paradigm. They are expected to form in the early Universe, in the course of multiple mergers of dark matter halos (hierarchical structure formation, [85]). SMBH are probably present in all (or almost all) galaxies [93,114] and their masses are correlated with the velocity dispersion in galactic bulges [44,51,114,171]. SMBH are closely related to just about every aspect of galactic activity, and may be one of the most significant energy sources in the Universe. Strength of the AGN activity depends critically on the dynamic properties of gas and stars in the nuclear region $[29,30,77]$, and may be connected to the presence of multiple SMBH in the host galaxy [103,153-155].

Most of the energy in the vicinity of SMBH is created through accretion, which ultimately releases the gravitational energy of infalling matter and pos- 
sibly mediates the process of releasing the rotational energy of the SMBH itself [18]. However, the dominating mechanism and detailed physics of the energy release are still not well understood - it could be the ionizing continuum from the accretion disk, the kinetic energy of the outflow [11], a magnetohydrodynamic mechanism $[122,123]$ or perhaps even a purely electromagnetic process [12]. A detailed picture of physical conditions in the immediate vicinity of SMBH is required for attempting to understand the mechanism for production, conversion and transport of the gravitational energy of the infalling material.

The most straightforward approach to studies of SMBH — probing their environment via direct imaging — is still out of reach because of extremely challenging requirements posed by this task on resolution and dynamic range of observations $[9,166]$. Direct detection and imaging of the event horizon of a black hole may become possible with the future interferometric missions planned in the X-ray [16,17] and radio [190] domains.

In this situation, physical properties of SMBH can be presently constrained from detailed studies of their environment on scales readily accessible for modern astrophysical instruments. Most important effects of SMBH on their environment are: 1) strong relativistic effects in the immediate vicinity of the event horizon, providing characteristic distortion of background line and continuum radiation $[9,27,28,82,188,166] ; 2)$ processing of the galactic gas and shaping the mass distribution and dynamics of gas and stars in the central regions of galaxies [77]; 3) energy and matter release into the ISM and IGM via outflows and emission (both substantially non-isotropic) during galaxy formation and evolution [25,68]; 4) stellar disruptions and other recurrent events of energy release (flares) $[63,134]$. Further detailed studies of these effects will be instrumental for understanding the physics of SMBH and their role in active galactic nuclei.

\section{Cosmological co-evolution of AGN and SMBH}

Recent years have seen an impressive progress in our understanding of cosmological co-evolution of active galaxies and supermassive black holes at their centres. The "demographics" of black holes can now be studied in detail, using the celebrated $M_{\mathrm{bh}}-\sigma_{\text {bulge }}$ relation $[89,171]$ and its derivatives connecting black hole masses in galaxies to observable properties of their nuclear emission. Evidence accumulates for SMBH to be residing practically in every galaxy with a nuclear bulge [61]. The black hole growth via accretion correlates with the bulge growth via star formation [85], which results in a tight correlation between the masses of bulges and central black holes [62]. The mass ratio $M_{\mathrm{bh}} / M_{\mathrm{bulge}}$ is likely to be higher at $z=2-3$, implying that black hole growth may induce the formation of galactic bulges. More massive galaxies and SMBH form at earlier cosmological epochs [65]. Initial assembly of SMBH in most powerful AGN begins at $z>10$ [15], and primordial SMBH in AGN are likely to contribute to reionization of the Universe [113]. The cosmological growth of SMBH is regulated by accretion, black hole mergers and stellar disruption events. The evolutionary sequence of AGN can be roughly outlined within four basic stages: manifestations 
of nuclear activity start from extremely obscured objects with a prominent submillimetre excess [111,124], then proceed to mainstream QSO stage, followed by an extremely red object (ERO) stage, and finally turning into "normal", "inactive" elliptical and spiral galaxies [23].

During the entire course of their evolution, AGN have a significant impact on the interstellar and intergalactic medium [25,38]. The radiative and kinetic feedback from AGN deposits vast quantities of energy into the environment on scales reaching several megaparsecs. The kinetic feedback from jets and BAL outflows reaches energies of $\sim 0.01 M_{\mathrm{bh}} c^{2}$ [69]. The radiative feedback influences strongly the SMBH growth in galaxies [25,164].

Galactic mergers and binary black holes are probably pivotal for the cosmological evolution of active galaxies. Powerful AGN are most likely produced in the course of galactic mergers [61], with every merger inducing two main episodes of accretion and star formation: at the first contact and at the final coalescence of two galaxies [25]. The nuclear activity is reduced when a loss cone is formed and most of nuclear gas is accreted onto SMBH [30,77], but it can be maintained at a relatively high level in the presence of a secondary black hole $[29,125]$. It is possible that the nuclear activity is closely related to dynamic evolution of binary SMBH in the centres of active galaxies [103]. Observational investigations of binary black hole systems in galaxies will be crucial for understanding the connection between SMBH and AGN.

\section{$6 \quad$ Fundamental questions}

Despite recent substantial advances in the field of AGN research, nuclear activity in galaxies still holds a number of puzzling questions that can only be answered with the next generation instruments which would provide orders of magnitude increases in sensitivity and resolution in just about every domain of astrophysical observations.

Accretion of galactic gas, stars, and (possibly) dark matter on supermassive black holes is now widely recognized as the most plausible mechanism of maintaining the black hole growth and nuclear activity in galaxies on cosmologically significant timescales. The gravitational potential energy of the infalling material and the rotational energy of the black hole are probably the primary sources of the AGN power. There is, however, a number of poorly understood fundamental issues connecting the observed manifestations of nuclear activity to the accretion process and physics of black holes. We still do not know the exact mechanism by which the gravitational and rotation energy is extracted and converted into emission and kinetic energy of relativistic plasma ejected from the nucleus. We need to understand how the mechanism and efficiency of the energy release is connected to the state of the SMBH (mass, spin, presence of a secondary $\mathrm{SMBH}$ in the nucleus) and physical conditions in the nuclear region. It is critical to understand whether and how these properties differ in the objects with and without powerful outflows. In a broader scope, putting these 
properties in the cosmological context would help attempting to reconstruct the pace of cosmological growth of SMBH and the evolution of their environment.

Resolving these fundamental issues should provide a sufficient basis for answering at least some of the "eternal" questions about galactic activity. Is there a unified model for all of the different types of AGN? Can we draw an evolutionary diagram for AGN? What is the relationship between the SMBH and the host galaxy? Do all galaxies have SMBH? Were black hole seeds or by-products of galaxy formation? How important is the feedback from supermassive black holes for the structure formation in the Universe? What is the role of accreting black holes in the reionization of the Universe? What causes the activity phase in a galaxy and sets its lifetime? How are AGN jets produced and collimated? How do they interact with, and affect, the host galaxy. How is the SMBH activity connected to galaxy mergers and central starbursts? Are multiple SMBH common in AGN, and how do they form and evolve? These are some of the problems that will hopefully be understood and resolved in the next decades with the help of the new generation of astrophysical facilities.

\section{References}

1. K. Abazajian, J.K. Adelman-McCarthy, M.A. Agüeros, et al.: AJ 126, 2081 (2003)

2. M.F. Aller, H.D. Aller, P.A. Hughes: ApJ 586, 33 (2003)

3. J.M. Anderson, J.S. Ulvestad, L.C. Ho: ApJ 603, 42 (2004)

4. R. Antonucci: Ann. Rev Astron. \& Astroph. 31, 473 (1993)

5. P. Arévalo, I. Papadakis, B. Kuhlbrodt, W. Brinkmann: A\&A 430, 435 (2005)

6. D.R. Ballantyne, S. Vaughan, A.C. Fabian: MNRAS 342, 239 (2003)

7. R.H. Becker, R.L. White, D.J. Helfand: ApJ 450, 559 (1995)

8. R.H. Becker, R.L. White, M.D. Gregg, et al.: ApJS 135, 227 (2001)

9. K. Beckwith, C. Done: MNRAS 359, 1217 (2005)

10. H.E. Bignall, D.L. Jauncey, L.L. Kedziora-Chudczer, et al.: PASA 19, 29 (2002)

11. R.D. Blandford, D.G. Payne: MNRAS 199, 883 (1982)

12. R.D. Blandford, R.L. Znajek: MNRAS 179, 433 (1976)

13. G.A. Bower, R.F. Green, A. Danks, et al.: ApJ 492, 111 (1998)

14. W. Brinkmann, P. Arévalo, M. Gliozzi, E. Ferrero: A\&A 415, 959 (2004)

15. V. Bromm, A. Loeb: ApJ 596, 34 (2003)

16. W. Cash: Adv. Space Res. 35, 122 (2005)

17. W. Cash, A. Shipley, S. Osterman, J. Marshall: Nature 407, 160 (2000)

18. M. Camenzind, R. Khanna: Il Nuovo Cimento 115 B, 815 (2000)

19. A. Caproni, Z. Abraham: ApJ 602, 625 (2004)

20. M.J. Claussen, P.J. Diamond, J.A. Braatz, A.S. Wilson, C. Henkel: ApJ 500, 129 (1998)

21. J.J. Condon, W.D. Cotton, E.W. Greisen, et al.: AJ 115, 1693 (1998)

22. T. Courvoisier: A\&A Reviews 9, 1 (1998)

23. L.Danese, F. Shankar, G.L. Granato, et al.: in Growing Black Holes: Accretion in a Cosmological Context, ESO Astrophysics Symposia Series, ed. S. Nayakshin, A. Merloni, R.A. Sunyaev (Springer-Verlag: Heidelberg 2005) p. 60

24. M. Dietrich, B.M. Peterson, P. Albrecht, et al.: ApJSS 115, 185 (1998)

25. T. Di Matteo, V. Springel, L. Hernquist: Nature 433, 604 (2005)

26. R. Dodson, P.G. Edwards, H. Hirabyashi: PASJ, subm. (2005) 
27. M. Dovčiak, S. Bianchi, M. Guainazzi, V. Karas, G. Matt: MNRAS 350, 745 (2004)

28. M. Dovčiak, V. Karas, G. Matt: MNRAS 355, 1005 (2004)

29. V.I. Dokuchaev: Sov. Astron. Lett. 15, 167 (1989)

30. V.I. Dokuchaev: MNRAS 251, 564 (1991)

31. A. Eckart, R. Genzel, T. Ott, R. Schödel: MNRAS 331, 917 (2002)

32. M. Elvis: ApJ 545, 63 (2000)

33. M. Eracleous, J.P. Halpern: ApJ 599, 886 (2003)

34. A.C. Fabian, K. Nandra, C.S. Reynolds, et al.: MNRAS 342, 1325 (1995)

35. A.C. Fabian: Proc. Natl. Acad. Sci. USA 96, 4749 (1999)

36. A.C. Fabian: astro-ph/0412224 (2004)

37. A.C. Fabian, S. Vaughan, K. Nandra, et al.: MNRAS 335, L1 (2002)

38. A.C. Fabian, J.S. Sanders, S.W. Allen, et al.: MNRAS 344, 43 (2003)

39. X. Fan, V.K. Narayan, M.A. Strauss, et al.: AJ 123, 1247 (2002)

40. X. Fan, M.A. Strauss, D.P. Schneider, et al.: AJ 125, 1649 (2003)

41. D. Fadda, B.T. Januzzi, A. Ford, L.S. Storie-Lombardi: AJ 128, 1 (2004)

42. H. Falcke, S. Markoff: A\&A 362, 113 (2000)

43. A.L. Fey, P. Charlot: ApJS 128, 17 (2000)

44. L. Ferrarese, D. Merritt: ApJ 539, L9 (2000)

45. A. Ferrari: ARA\&A 36, 539 (1998)

46. G.B. Field, R.D. Rogers: ApJ 403, 94 (1993)

47. E.B. Fomalont, S. Frey, Z. Paragi, et al.: ApJS, 131, 95 (2000)

48. G. Fossati, L. Maraschi, A. Celotti, A. Comastri, G. Ghisellini: MNRAS 299, 433 (1998)

49. S.C. Gallagher, W.N. Brandt, G. Chartas, G.P. Garmire, R.M. Sambruna: Adv. Space Res. 34, 2594 (2004)

50. J.F. Gallimore, S.A. Baum, C.P. O’Dea, E. Brinks, A. Pedlar: ApJ 462, 740 (1996)

51. K. Gebhardt, R. Bender, G. Bower, et al.: ApJ 539, L13 (2000)

52. R. Genzel, C.J. Cesarsky: Ann. Rev. Astron. \& Astroph. 38, 761 (2000)

53. M. Georganopoulos, A.P. Marscher: ApJ 506, 621 (1998)

54. A.M. Ghez, M. Morris, E.E. Becklin, A. Tanner, T. Kremenek: Nature 407, 349 (2000)

55. A.M. Ghez, S. Salim, S.D. Hornstein, et al.: ApJ 620, 744 (2005)

56. M. Giavalisco, H.C. Ferguson, A.M. Koekemoer, et al: ApJ 600, L93 (2004)

57. J.E. Greene, L.C. Ho: ApJ 610, 722 (2004)

58. M.D. Gregg, R.H. Becker, R.L. White, D.J. Helfand, R.G. McMahon, I.M. Hook: AJ 112, 407 (1996)

59. A.C. Gupta, D.P.K. Banerjee, N.M. Ashok, U.C. Joshi: A\&A 422, 505 (2004)

60. L.I. Gurvits, K.I. Kellermann, S. Frey: A\&A 342, 378 (1999)

61. M.G. Haehnelt, G. Kauffmann: MNRAS 318, 35 (2000)

62. N. Häring, H.-W. Rix: ApJ 604, 89 (2004)

63. J.P. Halpern, S. Gezari, S. Komossa: ApJ 604, 572 (2004)

64. M.C. Hartmann, M. Bötcher, G. Aldering, et al.: ApJ 553, 683 (2001)

65. G. Hasinger: in Growing Black Holes: Accretion in a Cosmological Context, ESO Astrophysics Symposia Series, ed. S.Nayakshin, A. Merloni, R.A. Sunyaev (Springer-Verlag: Heidelberg 2005) p. 418

66. T.M. Heckman, G. Kauffmann, J. Brinchman, S. Charlot, C. Tremonti, S.D.M. White: ApJ 613, 109 (2004)

67. J. Heidt, S.J. Wagner: A\&A 305, 42 (1996)

68. S. Heinz, R.A. Sunyaev: MNRAS 343, 59 (2003) 
69. S. Heinz, R.A. Sunyaev, A.Merloni, T. Di Matteo: Growing Black Holes: Accretion in a Cosmological Context, ESO Astrophysics Symposia Series, ed. S.Nayakshin, A. Merloni, R.A. Sunyaev (Springer-Verlag: Heidelberg 2005) p. 371

70. D.R. Henstock, I.W.A. Browne, P.N. Wilkinson, et al.: ApJS 100, 1 (1995)

71. J.R. Herrnstein, J.M. Moran, L.J. Greenhill, et al.: Nature 400, 539 (1999)

72. H. Hirabayashi, E.B. Fomalont, S. Horiuchi, J.E.J. Lovell, G.A. Moellenbrock: PASJ 52, 997 (2000)

73. L.C. Ho: in Observational Evidence for the Black Holes in the Universe, ed. S.K. Chakrabarti (Kluwer, Dordrecht 1999) p. 157

74. L.C. Ho: Adv. Space. Res. 23, 816 (1999)

75. L.C. Ho: ApJ 510, 631 (1999)

76. S. Horiuchi, E.B. Fomalont, W.K. Scott, et al.: ApJ, 616, 110 (2004)

77. P.B. Ivanov, A.G. Polnarev, P.Saha: MNRAS 358, 1361 (2005)

78. K. Iwasawa, A.C. Fabian, C. Reynolds, et al.: MNRAS 282, 1038 (1996)

79. W. Jaffe, K. Meisenheimer, H.J.A. Röttgering, et al.: Nature 429, 47 (2004)

80. W. Junor, J.A. Biretta, M. Livio: Nature 401, 891 (1999)

81. M. Kadler, E. Ros, A.P. Lobanov, H. Falcke, J.A. Lobanov: A\&A 426, 481 (2004)

82. V. Karas, D. Vokrouhlicky, A.G. Polnarev: MNRAS 259, 569 (1992)

83. S. Kaspi, P.S. Smith, H. Netzer, D. Maoz, B.T. Jannuzi, U. Giveon: ApJ 533, 631 (2000)

84. N.E. Kassim, R.A. Perley, W.S. Erikson, K.S. Dwarakanath: AJ 106, 2218 (1993)

85. G. Kauffmann, M.G. Haehnelt: MNRAS 311, 576 (2000)

86. K.I. Kellermann: PASA 19, 77 (2002)

87. K.I. Kellermann, M.L. Lister, D.C. Homan, et al.: AJ, 609, 539 (2004)

88. K.I. Kellermann, R.C. Vermeulen, J.A. Zensus, M.H. Cohen: AJ 115, 1295 (1998)

89. A. King: ApJ 593, 184 (2003)

90. H.-R. Klöckner, W.A. Baan: A\&A 419, 887 (2004)

91. H.R. Klöckner, W.A. Baan, M.A. Garrett: Nature, 421, 821 (2003)

92. S. Koide, K. Shibata, T. Kudoh, D.L. Meier: Science 295, 1688 (2002)

93. J. Kormendy, D.O. Richstone: Ann. Rev. Astron. \& Astroph. 33581 (1995)

94. Y. Kovalev, K.I. Kellermann, M.L. Lister, et al.: AJ 130, 2473 (2005)

95. A. Kraus, T.P. Krichbaum, R. Wegner, et al.: A\&A 401, 161 (2003)

96. T.P. Krichbaum, D. Graham, W. Alef, et al.: in New Developments in VLBI Science and Technology, ed. E. Ros R.W. Porcas, A.P. Lobanov, J.A. Zensus (MPIfR: Bonn 2002) p. 125

97. M.L. Lister: ApJ 599, 105 (2003)

98. M.L. Lister: astro-ph/0309413 (2003)

99. M.L. Lister, A.P. Marscher: Astroparticle Physics 11, 65 (1999)

100. M.L. Lister, P.S. Smith: ApJ 541, 66 (2000)

101. A.P. Lobanov: A\&A 390, 79 (1998)

102. A.P. Lobanov: A\&AS 132, 261 (1998)

103. A.P. Lobanov: in Growing Black Holes: Accretion in a Cosmological Context, ESO Astrophysics Symposia Series, ed. S.Nayakshin, A. Merloni, R.A. Sunyaev (Springer-Verlag: Heidelberg 2005) p. 354

104. A.P. Lobanov: MemSAItS 7, 12 (2005)

105. A.P. Lobanov, J. Roland: A\&A 431, 831 (2005)

106. A.P. Lobanov, J.A. Lobanov: ApJ 509, 521 (1999)

107. A.P. Lobanov, J.A. Lobanov: Science 294, 128 (2001)

108. A.P. Lobanov, P.E. Hardee, J.A. Eilek: New Astronomy 47, 629 (2003)

109. A.P. Lobanov, T.P. Krichbaum, D.A. Graham, et al.: A\&A 364, 391 (2000) 
110. C.J. Lonsdale, S.S. Doeleman, R.B. Phillips: AJ, 116, 8 (1998)

111. D. Lutz, E. Sturm, T. Alexander, et al.: in ISO Survey of a Dusty Universe, ed. D. Lemke, M. Stickel, K. Wilke, Lecture Notes in Physics, v. 548 (Springer-Verlag: Heidelberg 2000) p. 209

112. F. Macchetto, A. Marconi, D.J. Axon, A. Capetti, W. Sparks, P. Crane: ApJ 489, 579 (1997)

113. P. Madau, M.J. Rees, M. Volonteri, F. Haardt, S.P. Oh: ApJ 604, 484 (2004)

114. J. Magorrian, S. Tremaine, D.O. Richstone, et al: AJ 115, 2285 (1998)

115. A. Malizia, L. Bassani, J.B. Stephen, G. Malaguti, G.G.C. Palumbo: ApJS 113, 311 (1997)

116. L. Maraschi, P. Grandi, C.M. Urry, et al.: ApJ 435, 91 (1994)

117. A.P. Marscher, S.G. Jorstad, J.L. Gómez, et al.: Nature 417, 625 (2002)

118. H.L. Marshall, D.E. Harris, J.P. Grimes, et al.: ApJ 549, 167 (2001)

119. H.L. Marshall, B.P. Miller, D.S. Davis, et al.: ApJ 564, 683 (2002)

120. P. Marciano, J.W. Sulentic, R. Zamanov, et al.: ApJSS 149, 199 (2003)

121. G. Matt: Nuclear Physics B Proceedings Supplements 132, 97 (2004)

122. D.L. Meier: ApJ 548, 9 (2001)

123. D.L. Meier, S. Koide, Y. Uchida: Science 291, 84 (2001)

124. K. Menten, F. Bertoldi: Rev. Mod. Astron. 13, 229 (2000)

125. D. Merritt: in Coevolution of Black Holes and Galaxies, Carnegie Observatories Astrophysics Series, ed. L.C. Ho (CUP: Cambridge 2004) p. 264

126. G. Miniutti, A.C. Fabian, R. Goyder, A.N. Lasenby: MNRAS 344, 22 (2003)

127. M. Miyoshi, J. Moran, J. Herrnstein, et al.: Nature 373, 127 (1995)

128. C.G. Mundell, J.M. Wrobel, A. Pedlar, J.F. Gallimore: ApJ 583, 192 (2003)

129. N. Murray, J. Chiang: ApJ 474, 91 (1997)

130. R.F. Mushotzky, C. Done, K.A. Pounds: ARA\&A 31, 717 (1993)

131. K. Nandra, I.M. George, R.F. Mushotzky: ApJ 476, 70 (1997)

132. K. Nandra, I.M. George, R.F. Mushotzky: ApJ 477, 602 (1997)

133. S. Nayakshin: MNRAS 359, 545 (2005)

134. S. Nayakshin, J. Cuadra, R.A. Sunyaev: A\&A 413, 173 (2004)

135. P.M. Ogle, H.L. Marshall, J.C. Lee, C.R. Canizares: ApJ 545, L81 (2000)

136. F.N. Owen, P.E. Hardee, T.J. Cornwell: ApJ 340, 689 (1989)

137. T.J. Pearson, A.C.S. Readhead: ApJ 328, 114 (1988)

138. A.B. Peck, C. Henkel, J.S. Ulvestad, et al.: ApJ 590, 149 (2003)

139. E.S. Perlman, J.A. Biretta, F. Zhou, W.B. Sparks, F.D. Macchetto: AJ 117, 2185 (1999)

140. E.S. Perlman, W.B. Sparks, J. Radomski, et al.: ApJ 561, 51 (2001)

141. B.M. Peterson: in Advanced Lectures on the Starburst-AGN Connection, ed. by I.

Aretxaga, D. Kunth, R. Mújica. (World Scientific: Singapore, 2001), p. 3

142. B.M. Peterson, P. Berlind, R. Bertram, et al.: ApJ 581, 197 (2002)

143. A.G. Polatidis, P.N. Wilkinson, W. Xu., et al.: ApJS 98, 33 (1995)

144. G. Ponti, M. Cappi, M. Dadina, G. Malaguti: A\&A 417, 451 (2004)

145. L.Č. Popović, N. Stanić, A. Kubičela, E. Bon: A\&A 367, 780 (2001)

146. L.Č. Popović, E. Mediavilla, E. Bon, D. Ilić: A\&A 423, 909 (2004)

147. D. Proga, J.M. Stone, T.R. Kallman: ApJ 543, 686 (2000)

148. E.M. Puchnarewicz, K.O. Mason, F.J. Carrera: MNRAS 283, 1311 (1996)

149. F.T. Rantakyrö, L.B. Bååth, D.C. Backer, et al.: A\&AS 131, 451 (1998)

150. B.J. Rickett, L. Kedziora-Chudczer, D.L. Jauncey: ApJ 581, 103 (2002)

151. M. Ricotti, M.G. Haehnelt, M. Pettini, M.J. Rees: MNRAS 352, 21 (2004)

152. G.E. Romero, L. Chajet, Z. Abraham, J.H. Fan: A\&A 360, 57 (2000) 
153. N. Roos: A\&A 104, 218 (1981)

154. N. Roos: ApJ 294, 479 (1985)

155. N. Roos: ApJ 294, 486 (1985)

156. M. Salvati, M. Spada, F. Pacini: ApJ 495, 19 (1998)

157. R.M. Sambruna, I.M. George, R.F. Mushotsky, K. Nandra, T.J. Turner: ApJ 495, 749 (1998)

158. R.M. Sambruna, L. Maraschi, C.M. Urry: ApJ 463, 444 (1996)

159. A.I. Shapovalova, A.N. Burenkov, L. Carrasco, et al.: A\&A 376, 775 (2001)

160. D.C. Shih, K. Iwasawa, A.C. Fabian: MNRAS 333, 687 (2002)

161. P.A. Shaver, J.V. Wall, K.I. Kellermann, C.A. Jackson, M.R.S. Hawkins: Nature 384, 439 (1996)

162. M. Spada, M. Salvati, F. Pacini: ApJ 511, 136 (1999)

163. M. Spada, G. Ghisellini, D. Lazzati, A. Celotti: MNRAS 325, 1559 (2001)

164. V. Springel, T. Di Matteo, L. Hernquist: MNRAS 361, 776 (2005)

165. C.S. Stalin, Gopal Krishna, R. Sagar, P. Wiita: MNRAS 350, 175 (2004)

166. R. Takahashi: PASJ 57, 273 (2005)

167. Y. Tanaka, K. Nandra, A.C. Fabian, et al.: Nature 375, 695 (1995)

168. G.B. Taylor, R.C. Vermeulen, T.J. Pearson, et al.: ApJS 95, 345 (1994)

169. G.B. Taylor, R.C. Vermeulen, A.C.S. Readhead, et al.: ApJS 107, 37 (1996)

170. D.D. Thakkar, W. Xu, A.C.S. Readhead, et al.: ApJS 98, 33 (1995)

171. S. Tremaine, K. Gebhardt, R. Bender, et al.: ApJ 574, 740 (2002)

172. M. Türler, S. Paltani, T.J-L. Courvoisier, et al.: A\&AS 134, 89 (1999)

173. M. Türler, T.J-L. Courvoisier, S. Paltani: A\&A 361, 850 (2000)

174. M.H. Ulrich: Rev. Mod. Astron.: 5, 247 (1992)

175. M.H. Ulrich, L. Maraschi, C.M. Urry: ARA\&A 35, 445 (1997)

176. C.M. Urry, E.Treister: in Growing Black Holes: Accretion in a Cosmological Context, ESO Astrophysics Symposia Series, ed. S.Nayakshin, A. Merloni, R.A. Sunyaev (Springer-Verlag: Heidelberg 2005) p. 432

177. I.M. van Bemmel, C.P. Dullemond: A\&A 404, 1 (2003)

178. P. Véron, M.P. Véron-Cetty: Astron. \& Astroph. Rev. 10, 81 (2000)

179. B. Vollmer, T. Beckert, W.J. Duschl: A\&A 413, 949 (2004)

180. S.J. Wagner, A. Witzel: ARA\&A 33, 163 (1995)

181. W. Wamsteker, W. Ting-gui, N. Schartel, N., R. Vio: MNRAS 288, 225 (1997)

182. P.J. Wiita: astro-ph/0103020 (2001)

183. G. Weigelt, M. Wittkowski, Y.Y. Balega, et al.: A\&A 425, 77 (2004)

184. R.L. White, R.H. Becker, M.D. Gregg, et al.: ApJS 126, 133 (2000)

185. D. Worrall: MemSAIt 76, 28 (2005)

186. W. Xu, A.C.S. Readhead, T.J. Pearson, A.G. Polatidis, P.N. Wilkinson: ApJS 99, 297 (1995)

187. T. Yaqoob, I.M. George, K. Nandra, T.J. Turner, P.J. Serlemitsos, R.F. Mushotzky: ApJ 546, 759 (2000)

188. A.F. Zakharov, S.V. Repin: Adv. Space Res. 34, 2544 (2004)

189. A.F. Zakharov, N.S. Kardashev, V.N. Lukash, S.V. Repin: MNRAS 342, 1325 (2003)

190. A.F. Zakharov, A.A. Nucita, F. Depaolis, G. Ingrosso: New Astronomy 10, 479 (2005)

191. J.A. Lobanov: Ann. Rev. Astron. Astrophys. 35, 607 (1997)

192. J.A. Zensus, T.P. Krichbaum, A.P. Lobanov: Rev. Mod. Astron. 9, 241 (1996)

193. J.A. Zensus, E. Ros, K.I. Kellermann, M.H. Cohen, R.C. Vermeulen, M. Kadler: AJ 124, 662 (2002)

194. W. Zheng: AJ 111, 1498 (1996) 